\title{
Las elecciones federales del 2000, consolidación del panismo
}

De acuerdo con varios autores, el dos de julio del año 2000, por la vía electoral, pacíficamente, cayó uno de los regímenes autoritarios más longevos del mundo. ${ }^{1}$ Se cambiaron los referentes tradicionales de pensar y hacer en

Las elecciones federales del año 2000 significaron un cambio profundo en la estructura política y en el ánimo nacional.

El escrito ofrece los antecedentes electorales desde 1988. Establece una comparación entre las tres elecciones federales del 2 de julio. Hace un análisis del comportamiento de los electores jaliscienses desde el nivel de los distritos y de los municipios.Visualiza cómo desde el ámbito municipal se combinan los votos de castigo y los votos cruzados. Se hace un balance de las principales fuerzas electorales en Jalisco.

Ciesas 0 ccidente. docalons@ cencar.udg.mx política mexicana. Culminó la larga transición mexicana a la democracia. Para que ésta se consolide resta otro largo tramo, que incluye terminar con la desestructuración de los restos del partido de Estado, garantizar la plena libertad del voto, abrir las instancias gubernamentales al escrutinio de los ciudadanos, ciudadanizar muchas instituciones en donde el vie jo régimen tenía injerencia y control, pasar del combate a la pobreza como paliativo, a la participación social integradora, democratizar los medios masivos de comunicación, concluir con una reforma del Estado, etc. Muchas de las viejas reglas irán desapareciendo y se construirán nuevas. Esto iniciará otro proceso de transición. El presente escrito sólo intenta dar cuenta de cómo una fuerza electo-

1 César C ansino, La transición democrática 1997-2000, Cepcom, México, 2000. 
ral, la panista, se ha venido consolidando en J alisco. Se ofrece una visión nacional de las el ecciones federales del 2000 y una profundización en el casojalisciense.

Antecedentes electorales

Las elecciones presidenciales en 1988 habían mostrado una alta competitividad opositora. El partido del Estado tuvo que recurrir al fraude. Las cifras dadas como oficiales mostraban una caída del PRI del 70.9\% que había tenido en la elección de 1982, al 50.4\%. Seis años más tarde, en medio de un clima político convulsionado, el partido de Estado apenas pudo refrendar el $50.2 \%$ en unas elecciones muy inequitativas, apoyadas en financiamiento oculto que se convertiría después en un ingrediente importante del Fobaproa. Por su parte, el PAN había remontado un 17\% para al canzar un $26.7 \%$ en 1994 . Al principal contendiente de 1988, Cuauhtémoc Cárdenas, el régimen le reconoció un 30.9\% de los votos. Seis años después, en medio de un gran hostigamiento gubernamental, logró sól o un 17.1\%.

Las elecciones por las diputaciones federales entre 1979 y 1997 demostraron un sostenido auge opositor, con un bache en 1985 y un desplome de los índices del partido de Estado. La oposición en conjunto, de tener una cuarta parte de los votos, Ilegó a más de la mitad (ver cuadro 1). Como resultado de las elecciones de 1997, aunque el PRI mantenía una mayoría relativa en la Cámara de Diputados, la oposición unida lo superaba. La Cámara de Senadores, de ser casi monocolor a princi pios de los noventa, recibió un impulso pluripartidista en 1997 (ver cuadro 2).

En los congresos locales también se fue incrementando la representación de la oposición. Para 1999, el porcentaje de los diputados locales en todo el país era muy similar a la composición federal (PRI, 48.7\%; PAN, 26.8\%; PRD, 20.3\%; y otros, $4.2 \%)$. 
Cuadro 1. Porcentaje de diputados de 1979 a 1997 por los principales partidos

$\begin{array}{llrrrrr}\text { Año } & \text { PRI } & \text { PAN } & \text { PRD } & \text { PMS } & \text { PSUM } & \text { PCM } \\ 1979 & 74 & 10.8 & & & & 4.5 \\ 1982 & 74.8 & 12.8 & & & 4.3 & \\ 1985 & 73 & 9.5 & & & 3 & \\ 1988 & 52 & 20.2 & & 3.8 & & \\ 1991 & 64 & 17.8 & 8.2 & & & \\ 1994 & 60 & 23.8 & 14.2 & & & \\ 1997 & 47.8 & 24.2 & 25 & & & \end{array}$

Fuente: IFE

Cuadro 2. Porcentaje de senadores de 1991 a 1997

\begin{tabular}{rlrr} 
Año & PRI & PAN & PRD \\
1991 & 98.4 & 1.6 & \\
1994 & 74.2 & 19.5 & 6.3 \\
1997 & 60.2 & 25.8 & 12.5 \\
\hline
\end{tabular}

Fuente: IFE

La oposición libró una larga lucha en los municipios. Sin embargo, el recurso fraudulento por parte del partido de Estado hacía que los triunfos reconocidos fueran muy precarios. Los municipios gobernados por otro partido que no fuera el PRI, entre 1970 y 1978, al canzaron el número de 31. Entre 1979 y 1982 subieron a 60. De 1983 a 1989 llegaron a 114. En la década de los noventa, los triunfos opositores se incrementaron. En 1989, el PAN consiguió la primera gubernatura. Al iniciar el año 2000, el PAN gobernaba 7 estados, el PRD 4 y el PRI 21. EI PAN gobernaba en 12 capitales, el PRD en 6 y el PRI en 14. La mazorca del poder priista se iba desgranando. 


\section{Las elecciones federales del 2000}

Con las precampañas de los principales partidos, el tiempo electoral se alargó más de un año compl eto. Existía un ánimo mayoritario reflejado en encuestas que manifestaba el deseo de que la oposición se uniera para que el PRI dejara la presidencia de la República. Pese a intentos en este sentido, y a que lo más difícil se resolvió (el acuerdo sobre la plataforma), el mecanismo para decidir al candidato de una coalición opositora no pudo ser acordado. La alianza se frustró. Posteriormente se configuraron dos coaliciones en la oposición. La que configuró el PAN junto con el partido verde, que adoptó el nombre de Alianza por el Cambio. Su candidato fue Vicente Fox. Por su parte, el PRD conformó otra coalición con el PT y con tres de los pequeños partidos que acababan de obtener su registro: Convergencia por la Democracia (que correspondía a una escisión priista liderada por Dante Delgado), el Partido de la Sociedad Nacionalista (que respondía a intereses de un pequeño núcleo) y el Partido Alianza Social (en el que se encontraban restos de lo que había sido el partido sinarquista PDM). Esta coalición adoptó el nombre de Alianza por México. El candidato fue Cuauhtémoc Cárdenas. EI PRI, a finales de 1999, realizó una el ección abierta para determinar su candidato. Hubo una fuerte pelea entre el que había sido el Secretario de Gobernación, F rancisco Labastida, y el gobernador de Tabasco, Roberto Madrazo. EI aparato estatal se puso del lado del primero, quien ganó la nominación. Esta elección interna sirvió para impulsar la campaña del PRI. EI partido comandado por Gilberto Rincón Gallardo, Democracia Social, que había sido promovido por un subsecretario de Gobernación y que incorporó a antiguos salinistas, se lanzó con su líder como candidato presidencial. Manuel Camacho Solís, quien había formado el partido del Centro Democrático, fue postulado para contender por la presidencia del país. Finalmente, el muchas veces muerto y 
resucitado PARM Ianzó a Porfirio Muñoz Ledo como su candidato. Al final de la campaña, éste renuncióa la candidatura y se unió a la campaña de Fox. Así, al final Ilegaban seis partidos y coaliciones y cinco candidatos.

El monitoreo del comportamiento de los medios masivos de comunicación realizado por el IFE, difundido el 23 dejunio del 2000, reveló la falta de equidad en el tratamiento sobre Ios candidatos: al PRI se le dio un 39\%, a la Alianza por el Cambio un $27.3 \%$ y a la Alianza por México un $19.8 \%$. En cambio, el reparto del financiamiento público entre las tres principales fuerzas fue más parejo, pues Alianza por México tuvo el 34.1\%, Alianza por el Cambio el 30.2\%, y el PRI el $30.3 \%$.

La elección fue dominada por el marketing político. El escándalo como parte central de las contiendas se enseñoreó del ambiente político. Los ataques, sobre todo del sistema en contra del candidato de la Alianza por el Cambio, se incrementaron al final de la campaña.

La revisión de 47 encuestas electorales de noviembre de 1999 a junio del 2000 muestran que la mayoría de éstas, en lugar de dar certidumbre a los electores, se convirtieron en un instrumento más de campaña. Sobre todo, el gobierno presionó a medios de comunicación para que publicaran determinadas encuestas y dejaran de difundir otras. Aunque la mayoría daba como ganador al PRI, desde el principio hubo encuestas que anunciaban un triunfo opositor. ${ }^{2}$

2 En noviembre de 1999, Indemerc; en enero y abril del 2000, Gausscc; en febrero, marzo, abril, mayo y junio, G ea; en mayo y junio,Alducin; en junio, Dallas Morning. Rafael Giménez y Vidal Romero, en un artículo "Evaluación de las encuestas en la elección presidencial del 2000" (Este País, N úm. 114, septiembre 2000, Págs. 30-31), analizan las encuestas que fueron utilizadas como propaganda, las que se equivocaron de ganador y de estimaciones por partido, y las que acertaron en las proporciones dentro del margen de error en el ganador. Recalcan que los fracasos de Milenio y Reforma deberían ser revisados críticamente por los propios diarios.A puntan que uno de los problemas de las encuestas fallidas fue la selección de entrevistados. La utilización de filtros para seleccionar encuestados generó muestras sesgadas a favor del PRI en proporciones que llegaron a ser 
EI IFE hizo propaganda en contra de la coacción del voto. No obstante, la compra, coacción y condicionamiento del voto se hicieron presentes. El partido del Estado utilizó recursos públicos para beneficiarse, sobre todo los destinados al combate a la pobreza.

Alianza Cívica 2000 realizó un programa de observación en defensa del voto libre. Su evaluación de las elecciones federales del 2 de julio fue la siguiente: en un 3.6\% el presidente de la casilla no estaba en el encartado publicado por el organismo el ectoral; en un $22.6 \%$ de las casillas observadas, el elector no podía votar en secreto; en el $69.6 \%$ se presionóa varios para emitir su voto; y en un $14.1 \%$ se presionó a la mayoría; finalmente, en $\mathbf{1 3 . 8 \%}$ se detectaron algunas irregularidades.

Global Exchange, el 4 de julio, emitió un boletín de prensa en el que afirmaba que aunque el fraude electoral no había afectado los resultados de las el ecciones presidenciales, diversos incidentes de compra, coacción e intimidación se dieron en forma extensa en regiones del sur de México. Mexico Working Group hizo una declaración conjunta: Ia delegación de organizaciones no gubernamentales canadienses afirmó que las elecciones mexicanas tenían un avance significativo respecto al pasado, pero el fortalecimiento de la democracia permanecía como reto.

Aunque operadores del fraude en el PRI presionaban porque no se reconociera el triunfo de Fox, el Presidente de la República, basado tanto en encuestas de salida y conteos rápidos del mismo gobierno como en los contratados por el IFE, hizo el reconocimiento público de la victoria opositora. EI PRI tomó este anuncio como una afrenta. Sin embargo, la declaración presidencial dio certidumbre, y contra lo que no pocos esperaban, se inició un terso proceso de cambio de gobierno. 


\section{Los resultados}

La lista nominal era de 58'782,737. Votaron 37'603,523; el $63.97 \%{ }^{3}$ Se mejoró en casi dos puntos la participación de 1988; pero se quedó 13.8 puntos por debajo de la participación de 1994. Una de las explicaciones tiene que ver con la agresividad de las campañas que influyó en que se diera el caso de ciudadanos confundidos que prefirieron no acudir a las urnas.

Se trató prácticamente de un referéndum entre la continuidad y el cambio. Fox fue el candidato que convenció, sobre todo a los jóvenes, de que él abanderaba el cambio.

La elección presidencial sólo tuvo dos pequeñas impugnaciones. Alianza por México impugnó el ección de diputados en 17 distritos. Alianza por el Cambio en 3 (de Michoacán, ViIlahermosa y del D.F.) EI PRI impugnó en 11 distritos (en Aguascalientes, Baja California, Coahuila, J alisco, Michoacán, Sinaloa, Veracruz y Zacatecas). EI PRI impugnó además la elección de senadores en Colima; Alianza para el Cambio lo hizo en Yucatán. Estas impugnaciones las resolvió el tribunal y sól o hubo afectación en al gunas diputaciones.

El cuadro 3 ofrece los resultados el ectorales para Presidente de la República. Fox tuvo 1'192,911 votos menos que Zedillo seis años atrás. Labastida se quedó a 3'605,266 de la votación de Zedillo. Alianza por el Cambio (PAN y verde) ganó 14.8 puntos respecto a la elección del 1994. EI PRI perdió 14 puntos porcentuales. A Cárdenas en 1988 le fueron reconocidos 5'956,988 votos (31.1\%). En 1994 obtuvo 5'852,134 votos (17\%). En el año 2000, Cárdenas mejoró sus números absolutos, pero decreció porcentual mente. Hubo quienes interpretaron esto como un voto duro cardenista que había ll egado a un techo. Por su parte, el PRI en 1988 se adjudicó 9'641,329 votos (50.3\%), que incrementó a 17'181,625 (50.13\%) seis 
Cuadro 3. Resultados electorales en la elección presidencial del año 2000

Candidato

Fox

Labastida

Cárdenas

Rincón Gallardo

Camacho

PARM (sin candidato)

Nulos

Candidatos no registrados
Votos

$15^{\prime} 988,740$

13 '576,385

62259,048

592,075

208,261

157,119

789,838

32,457
Porcentaje

42.5

36.1

16.6

1.5

0.5

0.4

2.1

Fuente: IFE

años más tarde. En el 2000 decayó. El crecimiento del voto panista ha sido acelerado, pues de 3'267,159 (17\%) brincó a 9'146,841 (26.6\%) en 1994. En el 2000, junto con el partido verde consiguió un triunfo histórico.

En los estados gobernados por el PAN ganó F ox; pero también en 13 gobernados por el PRI y en el Distrito Federal gobernado por el PRD. En Baja California Sur, donde ganó una alianza opositora, también el voto mayoritario fue por Fox. Pero en Nayarit, Tlaxcala y Zacatecas, donde se había dado en recientes elecciones para gobernador el triunfo de coaliciones opositoras, el voto mayoritario fue para el PRI.

La mayor participación en la elección presidencial se dio en Yucatán con $71.95 \%$.

Siguieron el Distrito Federal (70.59), Querétaro (70.03), J alisco (68.19) y México (67.90). La menor participación fue en Chiapas (52.19), seguido de Guerrero (54.21).

En Baja California Sur, Ios el ectores dieron la victoria a Fox, pero hicieron triunfar al PRD en la elección para senadores. En Chiapas, Durango, Puebla, Quintana Roo, Tamaulipas, Veracruz y Yucatán también ganó F ox; pero para senadores, el triunfo fue para el PRI. En Michoacán, tanto en 
Cuadro 4. Elección para senadores en el año 2000

\begin{tabular}{lrc} 
Partidos o Coaliciones & \multicolumn{1}{c}{ Votos } & Porcentajes \\
Alianza por el Cambio & $14^{\prime} 215,252$ & 38.1 \\
PRI & $13^{\prime} 707,778$ & 36.7 \\
Alianza por México & $7^{\prime} 032,452$ & 18.8 \\
P. Democracia Social & 669,890 & 1.8 \\
P. Centro Democrático & 521,346 & 1.4 \\
PARM & 275,132 & 0.7 \\
No registrados & 31,080 & \\
Nulos & 852,627 & 2.2
\end{tabular}

la elección presidencial como en la de senadores, ganó el PRD.

Los resultados de la el ección para Senadores manifestó una importante variación comparada con la presidencial (ver cuadro 4). La distancia entre la Alianza para el Cambio y el PRI se acortó a menos de punto y medio porcentual. Hubo más votantes por senadores priistas que por el candidato presidencial de ese partido. Esto mismo ocurrió entre los votantes de Alianza por México. En 16 estados ganó el PRI, en 14 Alianza por el Cambio y en 2 Alianza por México. Las senadurías de primera minoría se distribuyeron 14 para el PRI, 11 para el PAN y 6 para el PRD.

En la elección de senadores hubo 298,366 votos menos que en la presidencial. Después de las resoluciones del Tribunal Electoral del Poder J udicial de la F ederación, los escaños se distribuyeron como se muestra en el cuadro 5. Aunque en números totales el PAN y el verde tenían más votos que el PRI, debido a que en esta elección el reparto se hace estado por estado, el resultado de escaños no corresponde matemáticamente a la cantidad de votos agregados nacionalmente. Con respecto a la anterior integración de la Cámara de Senadores, el PRI perdió 17 escaños, el PAN ganó 13 y el PRD ganó 2. 
Cuadro 5. Distribución en la Cámara de Senadores en el año 2000

\begin{tabular}{ccccc} 
Partido & Por mayoría & 1a minoría & Proporcional & Total \\
PAN & 27 & 10 & 9 & 46 \\
PRI & 32 & 15 & 13 & 60 \\
PRD & 4 & 7 & 4 & 15 \\
PV & 1 & & 4 & 5 \\
PT & & & 1 & 1 \\
Convergencia & & & 1 & 1 \\
\hline
\end{tabular}

Fuente: IFE

La elección de diputados se muestra en el cuadro 6. Los partidos coaligados en Alianza por el Cambio ganaron 5'337,341 votos en relación con la elección de tres años atrás. EI PRI mejoró en 2'307,043 votos. Los aliados PRD y PT perdieron 1'315,548 sufragios. EI PAN ganó 136 distritos uninominales; el PRI, 131; el PRD, 26; el PV, 5 y el PT, 2. Después de las resoluciones del Tribunal, las diputaciones se repartieron como se muestra en el cuadro 7. EI PRD perdió 75 diputados. La alianza que encabezó nole redituó votos y le mermó espacios y finanzas. De su votación del $18.8 \%$ le corresponden $3.6 \%$ al PT y $2 \%$ a cada uno de los otros tres coaligados. Por lo que le resta un $9.2 \%$ (menos de la mitad), lo que equivale a quince puntos menos que lo que había obtenido en 1997. EI PRI perdió 28 diputados. EI PAN ganó 85 y el verde $9 .{ }^{4}$

En la elección de diputados hubo 92,978 votos menos que en la de senadores y 391,344 menos que en la presidencial. Pero en 83 distritos, la elección de diputados fue superior a la de senadores (cuando debería ser igual o inferior).

4 C omo se puede ver en www.presidencia.gob.mx, al iniciar la LVII Legislatura (1997-2000), el PRI había alcanzado 165 triunfos en distritos y le fueron asignados 74 diputados plurinominales; el PRD ganó en 70 distritos y le fueron asignados 55 diputados plurinominales; el PAN ganó 64 distritos y le fueron asignados 57 diputados plurinominales; el PT ganó un distrito y se le asignaron 6 diputados plurinominales. Después, con el correr del tiempo hubo reacomodos en la Cá- 
Cuadro 6. Elección de diputados federales en el año 2000

\begin{tabular}{lrc} 
Partidos y Coaliciones & \multicolumn{1}{c}{ Votos } & Porcentaje \\
Alianza por el Cambio & $14^{\prime} 227,348$ & 38.2 \\
PRI & $13^{\prime} 734,140$ & 36.9 \\
Alianza por México & $66^{\prime} 954,016$ & 18.6 \\
P. Democracia Social & 699,152 & 1.8 \\
P. Centro Democrático & 428,927 & 1.1 \\
PARM & 272,635 & 0.7 \\
No registrados & 30,439 & 0.08 \\
Nulos & 865,930 & 2.3
\end{tabular}

Fuente: IFE

Cuadro 7. Distribución en la Cámara de Diputados

\begin{tabular}{lccc}
\multicolumn{1}{c}{ Partido } & Mayoría & Proporcional & Total \\
PAN & 136 & 70 & 206 \\
PRI & 132 & 79 & 211 \\
PRD & 24 & 26 & 50 \\
PV & 6 & 11 & 17 \\
PT & 1 & 6 & 7 \\
Convergencia & 1 & 3 & 4 \\
PSN & & 3 & 3 \\
PAS & & 2 & 2
\end{tabular}

Fuente: IFE

mara. El PRI perdió un diputado, pero después ganó 7, con lo que llegó a 245 diputados. El PRD fue perdiendo once y luego ganó uno, con lo que quedó con 115. El PAN perdió cuatro por lo que terminó con 117. Al PT se sumaron primero 6 y luego se retiró uno, con lo que quedó con 12. El verde perdió tres y terminó con 5 . Hubo un grupo parlamentario de cinco independientes. 
Cuadro 8. Porcentajes en el Congreso dela Unión

$\begin{array}{ccc}\text { Partidos } & \text { Diputados } & \text { Senadores } \\ \text { PAN } & 41.2 \% & 35.9 \% \\ \text { PRI } & 42.2 \% & 46.6 \% \\ \text { PRD } & 10.0 \% & 11.7 \% \\ \text { PV } & 3.4 \% & 3.9 \% \\ \text { PT } & 1.4 \% & 0.7 \% \\ \text { CD } & 0.8 \% & 0.7 \% \\ \text { PSN } & 0.6 \% & \\ \text { PAS } & 0.4 \% & \end{array}$

Fuente: IFE

Los porcentajes de puestos en el Congreso de la Unión quedaron como aparecen en el cuadro 8. Los partidos que conformaron la Alianza por México acumulaban un $13.2 \%$ en las diputaciones conseguidas, cuando habían tenido un 18.6\% de la elección. Estaban subrepresentados en 5.4 puntos. La Alianza por el Cambio sumaba $44.6 \%$ de representación frente a un $38.2 \%$ de votación. Tenía 6.4 puntos de sobrerrepresentación. EI PRI también tenía una sobrerrepresentación de 5.3 puntos. En el caso de los puestos en el Senado, la sobrerrepresentación del PRI era cercana a Ios diez puntos. Alianza por el Cambio llegaba a 39.8\%, con lo que superaba 1.7 puntos su porcentaje de votación. Alianza por M éxico tenía $13.1 \%$ de escaños frente a una votación de $18.8 \%$. Otra vez estaba por debajo 5.7 puntos.

Comparando las elecciones

En la elección presidencial de 1994, el PAN tuvo su mayor votación en la elección presidencial, seguido de la de senadores y en tercer sitio la de diputados. Para el PRI, Ia mejor votación fue la de senadores, seguida por la presidencial y en 
tercer sitio la de diputados. Para el PRD, su mejor votación fue la presidencial, le siguió la de senadores y en tercer lugar la de diputados.

En la elección del año 2000, Alianza por el Cambio tuvo su mejor votación en la presidencial, seguida por la de diputados y en tercer sitio la de senadores. EI PRI, a su vez, logrósu mejor votación en la de diputados, le siguió la de senadores y en tercer sitio la presidencial. Alianza por México consiguió su mejor votación en senadores, seguida de diputados y en último sitio la presidencial. Los partidos Democracia Social y Centro Democrático obtuvieron su mejor votación en la de senadores, después en la de diputados y en último sitio la presidencial.

Si calculamos el voto útil por Fox, éste se situó, al menos, en la cifra de 1'761,392 votos (un 11\% de su votación). Considerando la diferencia entre mejor votación de cada uno de los partidos y coaliciones y su votación presidencial, ese voto útil podría provenir de la siguiente forma: 773,404 de la Alianza por México (un $11 \%$ de su votación); 313,085 del Partido del Centro Democrático (el 60\% de su votación); 157,775 del PRI (1.1\% de su votación); 118,013 del PARM (42.9\% de su votación) y 77,077 del PSD (un 11\% de su votación). Alrededor de 23,692 ciudadanos votaron sól o por Presidente. Se pudo haber dado un voto útil que hubiera optado por los candidatos de Alianza por el Cambio en bloque; pero ése no se puede rastrear en las cifras electorales.

Se pueden apreciar otras diferencias. Así, 92,419 que votaron por senadores por el Partido del Centro Democrático, ya no lo hicieron por los diputados de ese partido (un 17.7\%); 78,436 que votaron por la Alianza por México en senadores tampoco repitieron ese voto en la elección de diputados (un $1.1 \%) ; 26,362$ que votaron por el PRI en diputados, no lo hicieron por el mismo partido en la elección de senadores (0.2\%); 12,096 que votaron por senadores de la Alianza por el Cambio no votaron de la misma forma para diputados (0.09\%). 
Final mente, 738 que votaron por senadores del PSD no sufragaron igual para diputados (0.1\%).

El cambio de votos de partido a partido llega a veces a implicar variaciones entre quienes aventajan en un distrito en una elección (presidencial) y los que la ganan en otra (diputados). En la elección de diputados, Alianza por el Cambio ganó 141 distritos, el PRI 131 y Alianza por México 28. Pero en la elección presidencial, Alianza por el Cambio tuvo mayoría de votos en 177 distritos, el PRI en 111 y Alianza por México sólo en 12.

En 1997, la votación favoreció al PRD en el Distrito Federal, Michoacán y Morelos; al PAN en Baja California, Colima, Guanajuato, J alisco, Nuevo León y Querétaro; en los 23 estados restantes, las cifras por el PRI estuvieron arriba. En la elección de diputados del 2000, Alianza por México (encabezada por el PRD) fue superior en los estados de Baja California Sur y Michoacán; el PAN en Aguascalientes, Baja California, Colima, Chihuahua, Distrito Federal, Guanajuato, J alisco, México, Morelos, Nuevo L eón, Querétaro, Quintana Roo, San Luis Potosí y Sonora; en los 16 restantes el PRI estuvo arriba.

Examinados 41 distritos en donde quien tuvo mayoría en la el ección presidencial no ganó la el ección de diputados, aparecen aspectos que conviene destacar. Esto sucede en 23 entidades federativas. En 16 casos, lo que pasó fue que la votación de Alianza por el Cambio bajó consi derablemente y subió la votación priista. En siete casos bajó Alianza por el Cambio y subió Alianza por México. En seis casos se invirtieron las cifras. Hubo casos en que la diferencia no fue grande. En otro, Alianza por México bajó y subió el PRI. Existieron diversas combinaciones: descendía uno mientras los otros dos incrementaban su votación; en esta división de ganancias, uno de ellos obtenía la diferencia ganadora.

Comparando los votos que al canzan los partidos y coaliciones en las elecciones de senadores y de diputados por en- 
tidad federativa aparece lo siguiente: Considerando la elección de diputados (en relación con la elección de senadores) en Coahuila, Guerrero, Nayarit y Tamaulipas, Alianza por el Cambio y Alianza por México bajan, el PRI sube. En Chiapas, Morelos, Quintana Roo y Tabasco, Alianza por el Cambio sube y Alianza por México y el PRI bajan. En Baja California Sur, Durango, México, Sinaloa y Sonora sube Alianza por México, bajan Alianza por el Cambio y el PRI. En Aguascalientes, Campeche y Distrito Federal, Alianza por el Cambio y Alianza por México suben, el PRI baja. En Colima, Michoacán, Tlaxcala, Veracruz y Zacatecas, Alianza por el Cambio y el PRI suben y Alianza por México baja. En Chihuahua, Guanajuato, J alisco, Nuevo León, San Luis Potosí, Hidalgo y Yucatán, Alianza por el Cambio baja, Alianza por México y el PRI suben. En Querétaro, Alianza por México permanece igual, Alianza por el Cambio baja, lo mismo que el PRI. En Baja California y Oaxaca, Ios tres bajan. En Puebla suben los tres.

Recapitulando las elecciones federales

Teniendo en cuenta sólo el financiamiento público de los partidos, ${ }^{5}$ cada voto de Alianza por el Cambio fue de 56.63 pesos; el del PRI costó 67.04 pesos; el costo del sufragio de Alianza por México se elevó hasta 163.68 pesos. Si se ve el total del financiamiento público a partidos y asociaciones y el gasto del IFE, cada voto promedió 224.80 pesos. Si sólo se contabiliza el financiamiento de los partidos, el costo del 2000 fue superior en trece veces al costo de 1994. Si al financiamiento público y al presupuesto del IFE seañade el financiamiento privado previsto legal mente (independientemente de lo que haya corrido debajo de la mesa), el costo del voto se eleva considerablemente.

5A lianza por el C ambio recibió 905'429,378.56;el PRI 910'241,014.88;yA lianza por México 1,024'478,700.48. 
Sergio Zermeño apuntó que los 30 distritos del Distrito Federal (el espacio de mayor concentración económica y cultural del país) fueron tricolores en 1994, amarillos en 1997 y azules en el $2000 .{ }^{6}$ De acuerdo con cálculos presentados por el periódico Reforma, ${ }^{7}$ el PRI perdió más de un millón de votos en zonas pobres. La revista Proceso ${ }^{\circ}$ consideró que los pobres de las ciudades votaron por Fox, y también al gunos pobres rurales. Hubo un voto diferenciado y regionalista. Muchos votantes estuvieron atentos más a candidatos que a partidos. Perduran importantes regiones donde campea el voto duro del PRI. El voto más pobre ha sido priista: el PRI ganó 95 de los 98 distritos rurales que había en 1994; y 80 de los 96 existentes en el 2000. El PAN ganó en el 2000 el $51 \%$ de los distritos con menos rezago. El voto perredista abarca sectores críticos y sectores medios relativamente escolarizados, sectores populares con alguna organización y fuerte exposición a los medios. También ha ido fraguando un voto duro.

Se dio un plebiscito por el cambio. Los electores diferenciaron mucho sus votos. Hubo el llamado voto útil a favor de Fox. Aunque en muchos distritos, sobre todo del sur del país y en zonas campesinas, se constató la compra y coacción del voto a favor del PRI, cosa que repercutió en varias diputaciones que ganó (ilegalmente) en esos sitios, la tendencia en el año 2000 fue a llevar a la presidencia a F ox, pero repartir entre las principales fuerzas las senadurías y diputaciones en tal forma que ningún partido por sí mismo tuviera mayoría absoluta ni pudiera decidir solo. Esto profundizó ya una tendencia que venía de 1997 en cuanto a un pluralismo que obliga a los partidos a tener que dialogar y a buscar consensos. Los partidos pequeños tuvieron diversa suerte. Los que se ampararon en coaliciones mantuvieron su registro. Los 
tres que contendieron solos no alcanzaron el porcentaje legal para refrendar su registro. La elección tuvo otro efecto importante en la vida política mexicana. Al perder el PRI la presidencia de la República se inicióla desestructuración del partido de Estado. Si ese partido remonta sus divisiones internas exacerbadas al haber perdido cabeza y rumbo, puede insertarse en un sistema de partidos ya sin las ventajas estatales que antes usurpaba. Por su parte, otro de los grandes perdedores ha sido el PRD. Las dos metas que le dieron nacimiento: acabar con el sistema de partido de Estado y llevar a Cárdenas a la presidencia con un programa de centro izquierda no las logró. Los electores le quitaron al PRI la presidencia, pero no por su medio. $Y$ el liderazgo cardenista se mermó. Ese liderazgo había logrado mantener una unidad en medio de fuertes pugnas internas. Ahora el PRD tendrá que buscar nuevos mecanismos para no desintegrarse. Fox de inmediato quiso alejarse del PAN para poder gobernar. Pero quedó tan acotado, que no podrá desligarse del partido por el cual llegó a la Presidencia.

Las elecciones federales en Jalisco

Campañas subsumidas

Las elecciones federales en J alisco estuvieron subordinadas al acontecer nacional. Las campañas más impactantes eran las de los candidatos presidenciales. Tuvo repercusión en los medios de comunicación el comportamiento y la campaña del candidato a Senador por el PRI en J alisco, el empresario tapatío ligado con el salinismo a través de Córdoba M ontoya, Raymundo Gómez Flores. Este personaje se había beneficiado de la privatización del Banco U nión, que después vendió a Cabal Peniche, y en el cual conservó un porcentaje de acciones. También había sido apuntado como uno de los empresarios que se aprovechó del F OBAPROA. I mitando cam- 
pañas del escándalo, privilegió un pleito en contra del Gobernador en el cual utilizó palabras vulgares e injuriosas. Sus actitudes prepotentes e intolerantes y su campaña de guerra sucia no le trajeron los dividendos que había calculado. Consiguió repudio hasta entre electores priistas.

Una encuesta del periódico Público a finales de mayo aseguraba que la intención del voto en J alisco en la elección presidencial apuntaba a que el PRI obtendría un 45\%, mientras el PAN Ilegaría a un 40\%. Por su parte, el periódico Mural publicaba días después que, en esa elección, el PAN alcanzaría al $44 \%$ mientras el PRI se quedaría con un 34\%. ${ }^{9}$

En junio, el gobernador solicitó públicamente que fueran removidos los delegados federales de Profeco y del I MSS porque hacían campaña a favor del PRI. En el periódico Público del 3 de agosto del 2000 apareció un reportaje en el que se demostraba que al PRI le había funcionado en J alisco el uso partidista de programas como Progresa. Por ejemplo, en comunidades indígenas de Poncitlán, los promotores de ese programa advertían a los beneficiarios que si perdía el PRI se acabaría ese beneficio. Lo mismo sucedió con los promotores de programas como Procampo y Crédito a la palabra que también manipularon dichos programas en beneficio del PRI. Hubo casillas en donde ese partido sacó hasta el 95\% de la votación. Alianza Cívica de J alisco denunció que un 7\% del padrón había sufrido acciones de compra de voto.

Para el 2000, el padrón fue de 3'900,016, y la lista nominal de 3'847,018. Hombres llegaban al $47.5 \%$ y mujeres al $52.4 \%$. El $80.9 \%$ eran originarios de J alisco; el 3.3\% de Michoacán, el $2.7 \%$ de Zacatecas, el 2.6 del D.F. y el $10.5 \%$ de otras entidades. Teniendo en cuenta las edades, el $4.8 \%$ correspondía a

9 La encuesta más errónea fue la de Público. N o sólo equivo có al ganador, sino que quedó 13 puntos por debajo en el caso de Fox y 9.1 puntos por arriba en el de Labastida. Mural apuntó bien quién llevaba la delantera en el ánimo de los electores, aunque anduvo 9 puntos por abajo. En lo concerniente al PRI se acercó bastante. 
Ios jóvenes entre 18 y 19 años; el 32.1\% estaba integrado por ciudadanos entre 20 y 29 años; un $23.8 \%$ pertenecía a los que tenían entre 30 y 39 años; el 16.5\% abarcaba a los que andaban entre 40 y 49 años; el 10.8\% comprendía a los de 50 y 59 años; de 60 años y más incluía un 11.9\%. Los distritos con mayor número de empadronados eran Tonalá y Tlaquepaque (246,725 y 244,381 , respectivamente). El menor era el de Colotlán (con 166,725). El promedio de empadronados fue de 205,264 ciudadanos.

Había 3,311 secciones. El distrito con mayor número de secciones era el XIX (con cabecera en Ciudad Guzmán), con 251; y el de menor número de secciones era el $X$ (con cabecera en Zapopan), con 109.

Se instalaron 6,968 casillas $(6,968$ básicas, 3,308 contiguas, 38 especiales y 148 extraordinarias). El 59.1\% estuvo en escuelas; el $5.7 \%$ en oficinas públicas, $8.6 \%$ en lugares públicos y el $26.4 \%$ en casas particulares. Sólo hubo cambios de funcionarios en la jornada el ectoral en $1.4 \%$ de las casillas (100) y únicamente se recurrió a una persona que estaba en la fila. Votó el 68\%. J alisco ocupó el cuarto lugar en participación en el país.

Los resultados de la elección

presidencial en Jalisco

Se esperaba que una gestión desaseada en el municipio de Zapopan disminuyera el voto panista; pero las elecciones federales en J alisco le dieron un importante repunte al panismo local. Los resultados de las elecciones federales en J alisco se encuentran en el cuadro 9. En la el ección presidencial, Alianza por el Cambio en J alisco consiguió 10.5 puntos más que su porcentaje nacional. EI PRI jalisciense se quedó en el nivel nacional. Pero Alianza por México se alejó 10.4 puntos abajo del porcentaje conseguido en todo el país. En la elección de Senadores, Alianza por el Cambio en J alisco re- 
Cuadro 9. Porcentajes de las tres elecciones federales en J alisco

$\begin{array}{lccc} & \text { Presidente } & \text { Senadores } & \text { Diputados } \\ \text { A. Cambio } & 53.07 & 49.54 & 49.28 \\ \text { PRI } & 35.90 & 35.14 & 36.04 \\ \text { A. México } & 6.23 & 7.07 & 7.96 \\ \text { PCD } & 0.97 & 3.42 & 1.72 \\ \text { PARM } & 0.42 & 0.64 & 0.73 \\ \text { DS } & 1.73 & 2.16 & 2.27 \\ \text { N/R } & 0.10 & 0.09 & 0.06 \\ \text { Nulos } & 1.87 & 1.95 & \end{array}$

montó 11.4 puntos el promedio de todos los estados; el PRI se puso abajo casi dos puntos; y Alianza por México se quedó 11.7 abajo. En la elección de diputados, Alianza por el Cambio en J alisco también remontó 10.9 puntos el porcentaje a nivel nacional; el PRI no se alejó del promedio conseguido en toda la república, pero el PRD siguió 10.7 puntos abajo. En J alisco, la distancia entre Alianza por el Cambio y el PRI fue de las más altas: 17 puntos en la presidencial, 14 en la de senadores y 13 en la de diputados.

Un $92.8 \%$ del voto por Fox provino de votantes que eligieron a Alianza por el Cambio en las tres opciones; 3.1\% de perredistas y $3.4 \%$ de los demás partidos. Pero leído desde otra óptica se puede ver que un $21 \%$ del voto perredista se fue a Fox; un $23 \%$ del voto del PSD, un $41.3 \%$ del voto parmista y el $60.4 \%$ del voto del PCD.

Un examen de las casillas para la elección presidencial en J alisco arroja lo siguiente: En el distrito I, el PRI gana el 72.4\% de las casillas; Alianza por el Cambio un 27.2\%. Hay una casilla empatada entre el PRI y Alianza por el Cambio. En el distritolI, el PRI gana el 18.5\% y Alianza por el Cambio el $89.9 \%$. Hay dos casillas empatadas entrePRI y Alianza por 
el Cambio. En el distrito III, el PRI gana el 9.7\%. Alianza por el Cambio gana el 90\%. Hay una casilla empatada entre PRI y Alianza por el Cambio. En el distritoIV, Alianza por México gana dos casillas y empata una con el PRI. EI PRI gana el 32.3\% y Alianza por el Cambio 66.5\%. En el distritoV, Alianza por México no obtiene ni un voto en cuatro casillas. EI PRI logra el triunfo en $39.9 \%$ de las casillas y Alianza por el Cambio en $59.2 \%$. Hay tres casillas empatadas entre el PRI y Alianza por el Cambio. En el distrito VI, el PRI lo hace en el $22.9 \%$ y Alianza por el Cambio en el $76.4 \%$. Hay dos casillas empatadas entre el PRI y Alianza por el Cambio. En el distrito VII, el PRI gana en 24.8\%; Alianza por el Cambio en 73.9\%. Hay cinco casillas empatadas entre el PRI y Alianza por el Cambio. En el distrito VIII, el PRI gana en 3.6\%; Alianza por el Cambio en $96.3 \%$. En el distrito IX, el PRI gana en $2.4 \%$ y Alianza por el Cambio en 97.5\%. En el distritox, el 100\% de Ias casillas las gana Alianza por el Cambio. En el distrito XI, el PRI gana el $4.4 \%$ de las casillas; Alianza por el Cambio el 95.3\%. En el distrito XII, el PRI sólo gana una casilla. EI 99.7\% de las casillas es ganado por Alianza por el Cambio. En el distrito XIII, el PRI gana el $1.1 \%$ de las casillas. EI 98.8\% es ganado por Alianza por el Cambio. En el distrito XIV, Alianza por México no obtiene ni un voto en una casilla. EI PRI gana en el 3.5\% y Alianza por el Cambio en el $96.4 \%$. En el distrito XV, Alianza por México gana dos casillas y empata otra con el PRI. En una más no logró ni un voto. EI $27.2 \%$ las gana el PRI y Alianza por el Cambio en $72.7 \%$. En una casilla, Alianza por el Cambio no tiene ni un voto. En el distritoXVI, el PRI gana el 13\% de las casillas y Alianza por el Cambio el $86.5 \%$. Hay dos empatadas entre el PRI y Alianza por el Cambio. En el distrito XVII, el PRI consigue ganar en $37.8 \%$ de las casillas; y Alianza por el Cambio en $61.3 \%$. Hay tres empatadas entre PRI y Alianza por el Cambio. En el distritoXVIII, Alianza por México gana el 3.8\% de las casillas el PRI el 59.5\% y Alianza por el Cambio el 35.8\%. Hay tres em- 
patadas entre el PRI y Alianza por el Cambio. En el distrito XIX, Alianza por México gana el $2.8 \%$ de las casillas; el PRI el 33.75 y Alianza por el Cambio el $63.1 \%$. Hubo una casilla empatada entre el PRI y Alianza por el Cambio. En dos casiIlas, Alianza por el Cambio no obtuvo ni un voto.

La diferencia de los totales (sin nulos) entre la votación presidencial en J al isco y la de diputados fue de 25,470 repartidos por distritos como se encuentra en el cuadro 10. En el caso del voto priista se votó más para presidente que para diputados $(5,186)$ de ese partido. Aunque en el caso de la elección para senadores fueron menos votos para esa opción que para diputados $(20,099)$. Pero hubo más votos para la elección de diputados que para la elección presidencial del PRI en 9 distritos (II, III, IV, V, VI, VII, XV, XVI, XIX).

Elecciones para Senadores

y Diputados en Jalisco

La diferencia entre la votación de senadores de Alianza por el Cambio y la de senadores del PRI en J alisco fue de 375,567. En la elección presidencial, la distancia a favor de Alianza por el Cambio es de 450,274 votos.

En el caso del PRD, en 17 distritos, la elección de diputados supera a la elección presidencial. Esto no acontece en los distritos XVI y XVII. Si bien, en todo el estado, la diferencia entre la elección presidencial del PRD y su votación por diputados favorece a esta última con distancia de 1.7 puntos porcentuales. La distancia mayor se encuentra en el distrito XVIII, donde la brecha es de 8.4 puntos.

Los senadores de Alianza por el Cambio al canzan 11,491 más votos que los diputados de esa coalición. En el PCD hay 44,753 más votos por senadores que por diputados. En los demás casos, la relación es inversa: en el PRI hay 20,099 votos menos en senadores que en diputados; en el PARM hay 2,264 votos menos y en el PSD hay 2,897 votos menos. 
Cuadro 10. Diferencias entrevotación presidencial por Fox y dediputados de Alianza por el Cambio en J alisco

$\begin{array}{lcc}\text { Distritos } & \text { Votos absol utos } & \text { Porcentajes (sin nulos) } \\ \text { I } & 3,059 & 2.5 \\ \text { II } & 5,313 & 3.4 \\ \text { III } & 3,294 & 2.1 \\ \text { IV } & 7,085 & 4.5 \\ \text { V } & 3,986 & 2.2 \\ \text { VI } & 4,871 & 3.3 \\ \text { VII } & 6,431 & 3.5 \\ \text { VIII } & 7,719 & 5.0 \\ \text { IX } & 4,634 & 3.2 \\ \text { X } & 9,137 & 6.0 \\ \text { XI } & 4,824 & 3.2 \\ \text { XII } & 8,349 & 5.1 \\ \text { XIII } & 6,349 & 4.1 \\ \text { XIV } & 4,872 & 3.5 \\ \text { XV } & 7,192 & 4.6 \\ \text { XVI } & 5,710 & 3.0 \\ \text { XVII } & 5,800 & 3.3 \\ \text { XVIII } & 8,261 & 6.5 \\ \text { XIX } & 4,547 & 3.0 \\ \text { Totales } & 111,433 & 3.8\end{array}$

La mejor votación del PCD en todo el país fue la de senadores en J alisco (3.4\%). Manuel Castelazo había sido candidato del PAN en la elección local de J alisco de 1997 en el distritox. Obtuvo 51,935 votos. En esa ocasión, ese candidato le implicó una pérdida de 11,315 votos a sólo cuatro meses de diferencia de la elección federal. En la elección del 2000, Castelazo se cambió de partido y jugó por la Senaduría por el PCD. En ese mismo distrito, en el año 2000, como candidato a 
senador por el PCD sacó 9,688 votos; cuatro veces menos que tres años antes.

Por estrecho margen, el PAN en 1994 había perdido la elección de Senadores. En el año 2000, los candidatos de Alianza por el Cambio ganaron hol gadamente. ${ }^{10}$

Los candidatos del PRI a las diputaciones uninominales fueron líderes agrarios de ese partido, líderes juveniles, diputados locales, ex diputados federales y locales, miembros de la CNOP, ex regidores, ex diputados locales y federales, funcionarios estatales y municipales, delegados de la SRA, miembros de la CNC. Los candidatos a diputados (por la vía de mayoría relativa) de la Alianza por el Cambio estaban relacionados con la estructura interna del PAN. Algunos tenían experiencia en municipios y pocos en materia legislativa.

En 1997, el PRI había triunfado en cuatro distritos: I, XV, XVIII y XIX. En el año 2000 sólo ganó tres: el V (Vallarta), el I (Colotán) y el XVIII (Autlán). ${ }^{11}$ Alianza por el Cambio ganó los otros 16 distritos. ${ }^{12}$

10 Llegaron al Senado por la vía del voto mayoritario los panistas FelipeVicencio y Gildardo Gómez Verónica. Por primera minoría alcanzó un escaño Raymundo Gómez Flores. Durante la campaña, en sus desplantes, había alardeado que si no ganaba, no aceptaría estar en el Senado por la vía de la primera minoría.Al concluir las elecciones se le preguntó si cumpliría su promesa. Prefirió el fuero, más en momentos en que la Suprema Corte ordenó al go bierno mexicano entregar al Congreso la documentación del Fobaproa, en donde podría salir implicado. Por la vía plurinominal,Tomás Vázquez, otro priista jalisciense, se integró al Senado.

11 En esta forma consiguió que fueran a la Cámara de Diputados Salvador Cosío (hijo del ex gobernador Guillermo Cosío Vidaurri), Jaime Hernández, dirigente de ejidatarios en Amatitlán, y Marcelo García, quien había sido subdirector de prestaciones económicas en el ISSSTE. Por la vía plurinominal llegaron a la Cámara los priistas jaliscienses José Manuel Correa Ceseña y J. Socorro Velázquez. El PRD también consiguió una curul en la Cámara de Diputados por la vía plurinominal. Le tocó a un integrante del grupo Universidad, J.A. Magallanes.

12 El PAN colocó en la Cámara de Diputados al ganadero José de Jesús Hurtado Torres; a José MaríaTejedaVázquez, que era diputado local y ejecutivo de la industria alimenticia; a EnriqueVilla Preciado, que había sido director de Patrimonio Municipal en el ayuntamiento de Zapopan; a Germán Pellegrini, que era el Secretario de juvenil de su partido; a Jaime Aceves Pérez, quien trabajaba en la CFE; a José A braham C isneros, ex regidor de Guadalajara y consejero estatal en su partido; aTomás Coronado 0 Imos, quien había estado en el ayuntamiento de 
Alianza por el Cambio impugnó el distrito V por compra del voto. EI PRI impugnótres: VI, XII y XVII. Las decisiones del Tribunal no modificaron la composición de los triunfos de los partidos en J alisco. En agosto del 2000 dejaron sus funciones en el Congreso de la Unión 32 diputados y 6 senadores jaliscienses. El primero de septiembre iniciaron sus trabajos legislativos 27 diputados por J alisco y 4 senadores.

Los resultados de la elección de diputados por distrito está en el cuadro 11. Los panistas, en relación con las elecciones de tres años atrás, triplicaron su distancia respecto del PRI. Este partido ha mantenido sus bastiones en los distritos I y XVIII, en los que reafirma una distancia arriba de los los diez puntos. En las el ecciones locales de 1997 y en las federales del 2000, el PRI ganó el distrito vallartensev; pero en ambas ocasiones lo hizo con estrecho margen. Por su parte, el PAN ganó otro distrito sól o con un punto de distancia: el XVII. Sólo en esos dos distritos hay una competencia cerrada. Por su parte, el PAN gana en 10 distritos con porcentajes arriba del $50 \%$ de los votos: Ios alteños y los de Guadal ajara, Tlaquepaque y dos de Zapopan. En uno de los zapopanos, la distancia de la votación panista con respecto de la priista es de 40 puntos. En el autlense distrito XVIII, el PRD consigue su mejor votación con un $22.7 \%$. este partido sólo en otros tres distritos supera los diez puntos. Democracia Social consigue más del 2\%. Sus mejores votaciones se encuentran en la zona metropolitana.

Yahualica; a Jorge Urdapilleta N úñez, ingeniero industrial, quien había sido diputado federal y dirigente en Zapopan; a Julio C ésar Lizárraga, ingeniero en computación y asesor del ayuntamiento de Guadalajara; a Miguel Ángel Martínez, quien había sido diputado federal y local, miembro del Consejo Político $\mathrm{N}$ acional de su partido; a Rodolfo 0 campo Velázquez, contador público; a Rodrigo Mileres, quien cursaba maestría en Finanzas; a Sergio García Sepúlveda, médico veterinario; a José Bañales $C$ astro, que había sido Vicepresidente en Tlaquepaque; a Rafael Ramírez Sánchez, quien había sido Presidente municipal deVilla Corona; y a Francisco Javier Flores $C$ hávez, ingeniero civil. Por la vía plurinominal también llegaron a la Cámara de Diputados los jaliscienses Herbert Taylor, quien había sido el Presidente estatal; Alonso Ulloa, que era diputado local; Antonio García, Secretario General en Jalisco, J.M. N úñez y Rocío García. 
Cuadro 11. Elección de diputados federales en J alisco. Absol utos y porcentajes (con nulos) 2000.

\begin{tabular}{lclllrc} 
Distrito & $\begin{array}{c}\text { Alianza por } \\
\text { d Cambio }\end{array}$ & $\%$ & PRI & \multicolumn{1}{c}{$\%$} & $\begin{array}{c}\text { Alianza por } \\
\text { México }\end{array}$ \\
I & 37,003 & 36.3 & 48,077 & 47.2 & 11,218 & 11 \\
II & 71,976 & 54.3 & 46,514 & 35.1 & 6,735 & 5 \\
III & 78,946 & 60 & 42,041 & 31.9 & 3,845 & 2.9 \\
IV & 65,185 & 44.2 & 59,581 & 40.4 & 11,329 & 7.7 \\
V & 50,291 & 42.8 & 51,718 & 44 & 10,262 & 8.7 \\
VI & 66,490 & 46.7 & 57,051 & 40.1 & 8,185 & 5.7 \\
VII & 76,664 & 47.3 & 64,014 & 39.5 & 10,047 & 6.2 \\
VIII & 78,652 & 53.9 & 45,947 & 31.5 & 9,738 & 6.6 \\
IX & 65,757 & 50.7 & 43,679 & 33.7 & 9,128 & 7 \\
X & 87,721 & 62.8 & 32,240 & 23.1 & 7,889 & 5.6 \\
XI & 69,203 & 51.4 & 45,351 & 33.7 & 9,351 & 6.9 \\
XII & 85,847 & 59.2 & 39,157 & 27 & 7,198 & 4.9 \\
XIII & 72,477 & 52.7 & 46,300 & 33.6 & 7,939 & 5.7 \\
XIV & 71,196 & 62.6 & 43,742 & 32.3 & 9,868 & 7.3 \\
XV & 63,754 & 47 & 49,644 & 36.6 & 16,639 & 12.2 \\
XVI & 82,137 & 50 & 59,202 & 36 & 10,560 & 6.4 \\
XVII & 61,645 & 42.8 & 60,247 & 41.8 & 14,107 & 9.7 \\
XVIII & 37,825 & 31.3 & 49,930 & 41.3 & 27,541 & 22.7 \\
XIX & 58,107 & 43.5 & 52,325 & 39.2 & 15,225 & 11.4
\end{tabular}

Las elecciones federales desde los municipios

El examen de la votación federal en los municipios nos ofrece importantes contrastes. En la elección para Presidente, el PRI fue el que tuvo más votos en 63 municipios; el PAN en 60 y el PRD en 1 . En la elección de diputados, el PRI aventajó en 68 municipios, el PRD en 3 y el PAN en 53. 
En la elección de senadores en el distrito I, un municipio donde el PRI había tenido una ligera ventaja en la presidencial, la pierde ante el PAN (Totatiche), donde curiosamente hay más votación para senadores que para presidente. En la elección de diputados vuelve a tomar ventaja el PRI. En el II distrito, en la elección presidencial y de senadores, el PRI sólo aventaja en Mexticacán; pero en la de diputados también lo hace en Acatic y en Yahualica.

En el DistritoXVII, en el municipio de Zacoalco, en la presidencial y en la de senadores aventaja el PAN; pero en la diputados el PRI, porque hubo un voto cruzado: electores que votaron por presidente y senadores panistas cambiaron en la de diputados para beneficiar al PRD, con lo que perdió ventaja el PAN frente al PRI.

En el Distrito XVIII, en Autlán aventajó el PAN en la elección de presidente y de senadores; pero en la de diputados aventaja el PRI, no porque haya incrementado sus votos, sino porque votos que habían sido para el PAN pasaron al PRD. En San Martín Hidalgo sucede lo mismo. En Casimiro Castillo, para las elecciones de presidente y de senadores, el PRI obtiene la delantera, pero en diputados, votantes panistas y priistas se van al PRD y este partido se pone al frente. Esto mismo sucede en EI Limón.

En el distrito XIX, el PRI aventajó para presidente en siete municipios, para senador en ocho y para diputados en nueve. En Mazamitla, el PAN estuvoal frente en la elección presidencial, pero perdió esa ventaja ante el PRI en las otras dos elecciones. En Pihuamo, el PAN estuvo arriba en la elección presidencial y desenadores, pero en la de diputados, votantes panistas votaron por el PRI y le dieron ventaja a este partido.

En 23 municipios gobernados por priistas (Arenal, Etzatlán, Encarnación, Lagos, San J ulián, Villa Hidalgo, ViIla Obregón, Ameca, J uanacatlán, EI Salto, Tonalá, Atotonilco, J amay, Ocotlán, Poncitlán, Amacueca, Chapala, Tlajomulco, Zacoalco, Gómez Farías, La Manzanilla, 
Tuxpan, Zapotiltic) ganó el PAN. Los votantes sancionaron a las administraciones priistas.

En los municipios panistas de Colotlán, Mexticacán, Atenguillo, Mascota, Atemajac, J ocotepec, Cuautla, J uchitlán, San Gabriel y Tecalitlán ganó el PRI. Los electores hicieron saber a estos diez ayuntamientos que no les estaba pareciendo su gestión.

EI PRD sól o tuvo ventaja en las tres elecciones en el municipio de Quitupan, del distrito XIX. En esta forma, en este municipio gobernado por el partido del sol azteca los votantes avalaron a esa administración. En Ahualulco de Mercado, en donde también había un ayuntamiento perredista, muchos votantes se pasaron al PRI, al que le dieron la delantera en las tres elecciones y en donde el PAN quedó en segundo sitio. Cocula era otro de los municipios perredistas. La elección del 2000 fue ganada ahí por el PAN; el PRD quedó en tercer lugar. Otro municipio en el que el PRD ha gobernado por tres períodos, Cuquío, la votación benefició al PRI. En la elección presidencial y de senadores, el PAN estuvo en segundo lugar, y ese sitio lo ocupó el PRD sólo en la el ección de diputados. Los perredistas estaban al frente del ayuntamiento deEI Limón. Ahí estuvieron en desventaja ante el PRI en las el ecciones presidencial y de senadores; pero mantuvieron la ventaja en la de di putados. En Mazamitla, otro de los municipios del PRD, este partido quedó en tercer sitio y perdió ya ante el PAN (elecciones presidenciales), ya ante el PRI (de senadores y diputados). En Ojuelos, el PRD había terminado con la hegemonía priista. En los comicios federales del 2000 cayó al tercer sitio y los votantes de ese municipio prefirieron al PAN. En San Marcos, el PRD vencióal PRI en 1997. En el 2000, el tricolor volvió a tomar ventaja y el perredismo bajó hasta el tercer sitio. En Sayula, municipio perredista, el PAN tomó la delantera y el PRD pasó al tercer lugar. Finalmente, en Tala, la votación perredista queda muy lejana del PRI (que aventajó en las tres elecciones) y del PAN (que se 
puso en segundo sitio). Ahí hubo votantes perredistas que prefirieron a Fox. En Tomatlán, el PRD bajó al segundo sitioy perdió ante el PRI. Pero en el municipio priista Casimiro Castillo, el PRD aventajó en la elección de diputados.

En Acatlán de J uárez, la administración del partido verde no fue avalada y el PRI tuvo la ventaja. En Cabo Corrientes, el PT tampoco fue refrendado y la mayoría la tuvo el PRI. Lo mismo le sucedió en Tuxcueca, donde la votación favoreció al PAN.

Una visión de nueve elecciones

Una visión del comportamiento el ectoral en las últimas nueve elecciones (locales y federales) se puede apreciar en el cuadro 12. EI PRI había tenido un serio descalabro en las elecciones federales de 1988. I nició una recuperación en las locales de ese año, para alcanzar un alto nivel en las federales de 1991 (apoyado en el Programa Solidaridad). Declinó un poco en las locales del 92. En 1994, aunque superó el miIlón de votos, sus porcentajes decayeron. En la local de 1994 sufrió un grave tropiezo. Cayó aún más en la federal del 97, en la que obtuvo su menor porcentaje. En la local de ese mismo año mantuvo números absolutos y relativos. Atrajo nuevos votantes en la federal del 2000, pero sus porcentajes siguieron en los niveles de los últimos cinco años.

EI PAN había conseguido importantes triunfos en el 88. Dedinó en las siguientes dos el ecciones pero se recuperó en el 91. En la federal de 1994 remontó en números absolutos de votos y en su porcentaje. En 1995 superó el millón de votantes y traspasó la cifra del $50 \%$. Perdió votantes y porcentajes en las dos elecciones del 97. En la del 2000 superó los números absolutos del 95 y se volvió a colocar muy cercano del $50 \%$.

El cardenismo en J alisco irrumpió en el 88. Después sufrió graves pérdidas de votantes, con leves reajustes entre la local del 88 y la del 95. En las elecciones del 97 tuvo impor- 


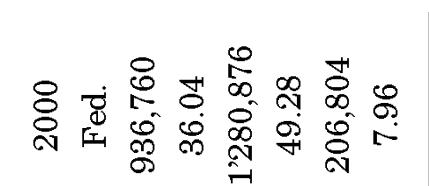

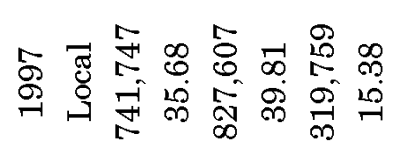

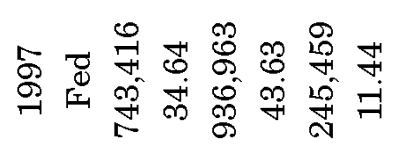

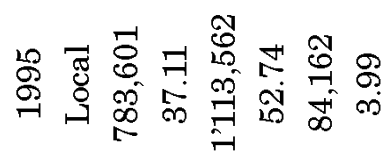

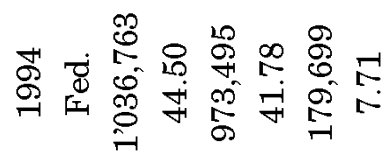

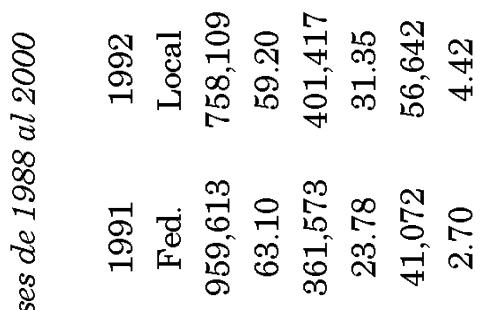

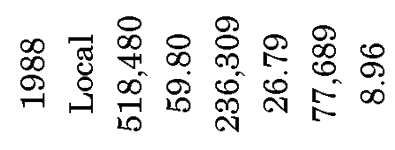

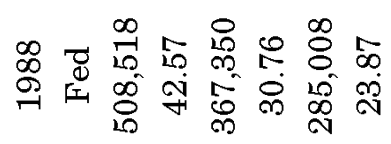

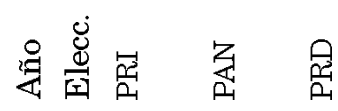

tante repunte pero en el 2000 perdió votos absolutos y la mitad de su porcentaje.

Recapitulando las elecciones federales del 2000 en Jalisco

Para todo el país, las elecciones federales del año 2000 implicaron un importante cambio político. La derrota del PRI en la elección presidencial abrió una nueva era política para la nación. Se al entó la esperanza de cambi os importantes. Había hastío de la corrupción, del corporativismo, del autoritarismo, del centralismo, del elitismo tecnocrático, de que los procesos democráticos fueran trampeados, de la falta de respeto a la libertad del voto, de que no hubiera transparencia en las acciones de gobierno, de la ausencia de canales de participación desde abajo. La sola derrota priista no implicaba necesariamente que se conjuraran todos esos males, pero se abría la puerta para ventilar un ambiente muy viciado. La pieza clave del partido de Es- 
tado, el presidencialismo priista, había desaparecido. Los restos de ese sistema de partido se mantenían con los gobernadores priistas y en una correosa cultura política configurada por las formas de hacer política que ese partido había introyectado en amplias capas sociales. Aun los partidos opositores se habían Ilegado a contaminar de esa cultura. En un estado gobernado hacía más de cinco años por el PAN, se ganaba que las dependencias federales en la entidad ya no fueran usadas electoralmente a favor del PRI, aunque todavía permanecieran cotos del corporativismo priista en varios sindicatos. Hubo una sensación de victoria ciudadana. Hacía mucho tiempo que las victorias presidenciales del PRI eran simplemente asumidas, pero no festejadas. EI triunfo de Fox fue cel ebrado ruidosamente en las calles.

EI PAN se consolidó. En más de la mitad de los distritos, sus índices van del $50 \%$ de los votos al $63 \%$. Este núcleo se encuentra en las zonas urbanas con mayores índices económicos. Regional mente se ubica en la zona metropolitana y en Los Altos. En siete distritos, su votación va del $40 \%$ al $49 \%$. Su más baja votación (entre 30 y 37\%) está en la zona rural norteña y en la que encabeza Autlán. Por su parte, el PRI se mantiene como una importante segunda fuerza política en $\mathrm{J}$ alisco. Ha logrado mantener un voto duro al rededor del $35 \%$ de los votos. Su fortaleza es principal mente rural. Ahí se ubica una tercera parte de los distritos en los que va del $40 \%$ al $47 \%$. En más de la mitad de los distritos, sus votos oscilan entre un 30 y 39\%. Hay dos zonas urbanas en donde su votación se ha deteriorado (27 y 23\%). EI PRD se encuentra muy lejos de estos dos partidos. Ha decaído por debajo de los 10 puntos. Pero en el distrito con cabecera en Autlán se ha consolidado como tercera fuerza con cerca de una cuarta parte de los votantes. Lo más destacado del comportamiento de los electores jaliscienses en los comicios federales del 2000 ha sido la capacidad de utilizar un voto diferenciado. Es notable el voto de castigo ahí donde mandan señales de desaproba- 
ción a autoridades municipales. Aun en zonas rurales depauperadas hay ciudadanos que hacen uso del voto cruzado por distintos partidos dependiendo de las coyunturales locales y de aspiraciones en relación con lo nacional. Pese al voto todavía comprado, obligado y aun corporado hay indicios de un ejercicio libre y responsable del voto frente a los partidos. Esto indica que hay un crecimiento de la valoración del voto y de la capacidad ciudadana. $\mathbf{E}$ 